\title{
Classification of endometriosis
}

\author{
Soo-Young Lee ${ }^{1}$, Yu-Jin Koo ${ }^{2}$, Dae-Hyung Lee ${ }^{2}$ \\ ${ }^{1}$ Department of Obstetrics and Gynecology, Yeungnam University Hospital, Daegu, Korea \\ ${ }^{2}$ Department of Obstetrics and Gynecology, Yeungnam University College of Medicine, Daegu, Korea
}

Received: June 9, 2020

Revised: July 9, 2020

Accepted: July 16, 2020

Corresponding author:

Yu-Jin Koo

Department of Obstetrics and

Gynecology, Yeungnam University

College of Medicine, 170

Hyeonchung-ro, Nam-gu, Daegu

42415, Korea

Tel: +82-53-620-3433

Fax: +82-53-654-0676

E-mail:yujinkoo@yu.ac.kr
Endometriosis is a chronic disease associated with pelvic pain and infertility. Several classification systems for the severity of endometriosis have been proposed. Of these, the revised American Society for Reproductive Medicine classification is the most well-known. The ENZIAN classification was developed to classify deep infiltrating endometriosis and focused on the retroperitoneal structures. The endometriosis fertility index was developed to predict the fertility outcomes in patients who underwent surgery for endometriosis. Finally, the American Association of Gynecological Laparoscopists classification is currently being developed, for which 30 endometriosis experts are analyzing and researching data by assigning scores to categories considered important; however, it has not yet been fully validated and published. Currently, none of the classification systems are considered the gold standard. In this article, we review the classification systems, identify their pros and cons, and discuss what improvements need to be made to each system in the future.

Keywords: American Society for Reproductive Medicine classification; Classification; Endometriosis; ENZIAN classification

\section{Introduction}

Endometriosis is a chronic inflammatory disease that can cause pelvic pain and infertility. It usually affects $2 \%$ to $10 \%$ of women of reproductive age [1]. In 2010, the prevalence of endometriosis in women aged 15 to 49 years was about 1.7 billion worldwide [2]. According to a survey conducted by Statistics Korea, the number of patients who visited the hospital for endometriosis was about 100,000 in 2016 and 120,000 in 2018, and is gradually increasing [3]. With the rising trend of endometriosis, medical expenses are also likely to increase. The annual economic burden of endometriosis, including direct health care costs and indirect productivity loss, was estimated to be 22 billion US dollar (USD) in 2002 and 69.4 billion USD in 2009 in the United States of America [4].

The lack of a gold standard staging system is a concerning issue in the treatment of endometriosis. A reproducible and well-organized classification system is necessary not only to clarify communication among physicians but also to standardize the optimal treatment strategy. Sampson [5] first classified endometriosis by describing ovarian hemorrhagic cysts and their associated adhesion formation in 1921. He classified ovarian hematomas into four subgroups: follicular, corpus luteal, stromal, and endometrial. However, no optimal classification system currently exists, and the usefulness of the current classification systems is controversial. The clinical manifestations of endometriosis are diverse, and the relationship between symptom and disease severities is ambiguous. So far, many efforts have been made to better classify endometriosis. The ideal classification system should be able to explain the extent of disease, predict pain and fertility, provide accurate information to patients, and reflect the anatomical features. This article reviews the four standard classification systems, namely, the revised American Society for Reproductive Medicine (rASRM) classification, ENZIAN classification, endometriosis fertility index (EFI), and American Association of Gynecological Laparoscopists (AAGL) classification.

Copyright (C) 2021 Yeungnam University College of Medicine

This is an Open Access article distributed under the terms of the Creative Commons Attribution Non-Commercial License (http://creativecommons.org/licenses/by-nc/4.0/) which permits unrestricted non-commercial use, distribution, and reproduction in any medium, provided the original work is properly cited. 


\section{American Society for Reproductive Medicine classification}

The American Fertility Society (AFS) proposed a unique approach, the AFS score, in 1979 [6]. The stage of endometriosis was derived from a cumulative score. The weighted value system was scored and summed according to the size of the endometriotic lesions in the ovaries, peritoneum, and fallopian tubes, and the severity of adhesion at each of the aforementioned sites. The staging system was divided into four stages: I ( 1 to 5 points, mild), II (6 to 15 points, moderate), III (16 to 30 points, severe), and IV (31 to 54 points, extensive). However, some critics have indicated a problem with this classification system. Hasson [7], for example, pointed out the lack of a relationship between the disease stage and the clinical symptoms of pain and infertility. Therefore, this system was revised in 1985 (Fig. 1). They defined the stage classification as minimal, mild, moderate, and severe, and each score was reclassified as 1 to 5,6 to 15,16 to 40 , and more than 40 . Tubal endometriosis was omitted from the revised classification, and the lesions of endometriosis were classified as superficial and deep lesions. The size of deep ovarian endometriosis $>3 \mathrm{~cm}$ scored 20 points, and dense ovarian adhesion and dense tubal blockage were adjusted upward to 16 points. In addition, a single finding of complete cul-de-sac obliteration scored 40 points and was classified as severe disease. In 1996, this scoring system was renamed as the revised American Society for Reproductive Medicine (rAS$\mathrm{RM})$ classification [8].

One of the advantages of the rASRM classification is that it has been accepted globally and has been widely used in recent years. In addition, it is easy to use and helpful for physicians to explain the degree of endometriosis in simple terms to patients. However, there are several disadvantages. First, there is a difference between histologically diagnosed endometriosis and visually diagnosed stage. Fernando et al. [9] compared the pathologic findings of the surgically removed endometriosis and visually diagnosed the rASRM stage. The rASRM stages I to IV were concordant in $49.7 \%$, $80.3 \%, 78.1 \%$, and $78.9 \%$ of the patients, respectively, indicating that women with rASRM stage I disease are more likely to be misdiagnosed as having endometriosis on visual inspection. Second, the reproducibility of the rASRM score is poor. Hornstein et al. [10] reported that comparison of interobserver and intraobserver scores resulted in a change in endometriosis stage in 52\% and 38\% of the patients, respectively. Third, severities of pain and infertility are not correlated with rASRM stage. In a study of 244 patients, Vercellini et al. [11] reported that endometriosis stage was not consistently related to pain symptoms, and that only the presence of vaginal lesions was frequently associated with severe deep dys- pareunia. In addition, according to Guzick et al. [12], there was a slight decrease in the pregnancy rate in stage IV endometriosis, but no significant difference in pregnancy rates between different stages was found. Fourth, rASRM classification does not consider the presence of deeply infiltrating endometriosis (DIE) in different sites such as the uterosacral ligaments, bladder, vagina, and bowel. Therefore, in order to supplement DIE in retroperitoneal structures, the ENZIAN classification was developed.

\section{ENZIAN classification}

The ENZIAN classification was introduced in Austria in 2005 [13]. The ENZIAN score, like the rASRM classification, is determined by the extent of endometriosis during surgery. When the ENZIAN classification was first developed, its purpose was not to compete with the rASRM classification, but to supplement it with respect to the description of DIE. However, according to Haas et al. [14], there was unintended partial overlapping with the rASRM score. Fifty-eight of 160 patients with solitary superficial peritoneal disease in the pouch of Douglas cavity, uterosacral ligament, or a combination of the two, were classified according to ENZIAN, although they did not fulfill the criteria of DIE and had previously been classified according to the rASRM classification. Therefore, two revisions of the ENZIAN classification system were carried out in 2010 and 2011 to correct the overlap between rASRM and ENZIAN systems and to make it easier to use (Fig. 2) $[15,16]$. The revised ENZIAN classification was simplified by dividing retroperitoneal structures into three compartments. The posterior part of the uterus was divided into compartment A consisting of the rectovaginal septum and vagina, compartment $B$ consisting of the uterosacral ligament and pelvic walls, and compartment $\mathrm{C}$ consisting of the sigmoid colon and rectum. The severity of the lesion is set to invasiveness $<1 \mathrm{~cm}$ for grade 1 , invasiveness 1 to $3 \mathrm{~cm}$ for grade 2 , and invasiveness $>3 \mathrm{~cm}$ for grade 3 . The prefix "E" indicates the presence of a tumor of endometriosis. The number that follows the prefix indicates the size of the lesion, and after the number, the lowercase English letter indicates the affected compartment. Two lowercase English letters mean bilateral disease. The invasion of endometriosis to other organs in the pelvic cavity and to distant organs is expressed as follows: "FA" is defined as adenomyosis, "FB" as involvement of the bladder, "FU" as intrinsic ureter involvement, "FO" as involvement of other locations, and "FI" as intestinal involvement. This revision is helpful for physicians to better understand and readily use the ENZIAN classification.

One of the advantages of the ENZIAN classification is that it provides detailed descriptions of the retroperitoneal structures. 


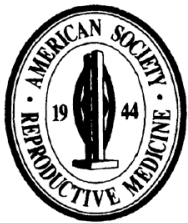

\section{AMERICAN SOCIETY FOR REPRODUCTIVE MEDICINE REVISED CLASSIFICATION OF ENDOMETRIOSIS}

\begin{tabular}{|c|c|c|c|c|}
\hline \multicolumn{3}{|c|}{ Patient's Name } & \multicolumn{2}{|l|}{ Date } \\
\hline \multirow{2}{*}{\multicolumn{2}{|c|}{$\begin{array}{ll}\text { Stage I (Minimal) } & -1-5 \\
\text { Stage II (Mild) } & -6-15 \\
\text { Stage III (Moderate) } & -16-40 \\
\text { Stage IV (Severe) } & ->40 \\
\text { Total }\end{array}$}} & $\begin{array}{l}\text { Laparoscopy } \\
\text { Recommended Treatme }\end{array}$ & \multicolumn{2}{|c|}{ Photography } \\
\hline & & \multicolumn{2}{|l|}{ Prognosis } & \\
\hline \multirow{3}{*}{ 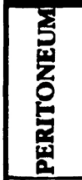 } & ENDOMETRIOSIS & $<1 \mathrm{~cm}$ & $1-3 \mathrm{~cm}$ & $>3 \mathrm{~cm}$ \\
\hline & Superficial & 1 & 2 & 4 \\
\hline & Deep & 2 & 4 & 6 \\
\hline \multirow{6}{*}{ z) } & R Superficial & 1 & 2 & 4 \\
\hline & Deep & 4 & 16 & 20 \\
\hline & L Superficial & 1 & 2 & 4 \\
\hline & Deep & 4 & 16 & 20 \\
\hline & \multirow{2}{*}{\begin{tabular}{|l|} 
POSTERIOR \\
CULDESAC \\
OBLTTERATION
\end{tabular}} & \multicolumn{2}{|l|}{ Partial } & Complete \\
\hline & & 4 & & 40 \\
\hline \multirow{5}{*}{ 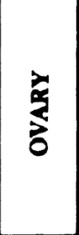 } & ADHESIONS & $<1 / 3$ Enclosure & 1/3-2/3 Enclosure & $>2 / 3$ Enclosure \\
\hline & R Filmy & 1 & 2 & 4 \\
\hline & Dense & 4 & 8 & 16 \\
\hline & L Filmy & 1 & 2 & 4 \\
\hline & Dense & 4 & 8 & 16 \\
\hline \multirow{4}{*}{ 狊 } & R Filmy & 1 & 2 & 4 \\
\hline & Dense & $4^{\circ}$ & $8^{\circ}$ & 16 \\
\hline & L Filmy & 1 & 2 & 4 \\
\hline & Dense & $4^{\circ}$ & $8^{\circ}$ & 16 \\
\hline
\end{tabular}

-If the fimbriated end of the fallopian tube is completely enclosed, change the point assignment to 16 .

Denote appearance of superficial implant types as red [(R), red, red-pink, flamelike, vesicular blobs, clear vesicles], white [(W), opacifications, peritoneal defects, yellow-brown], or black [(B) black, hemosiderin deposits, blue]. Denote percent of total

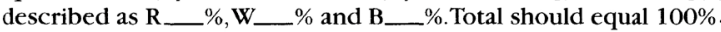

Additional Endometriosis:

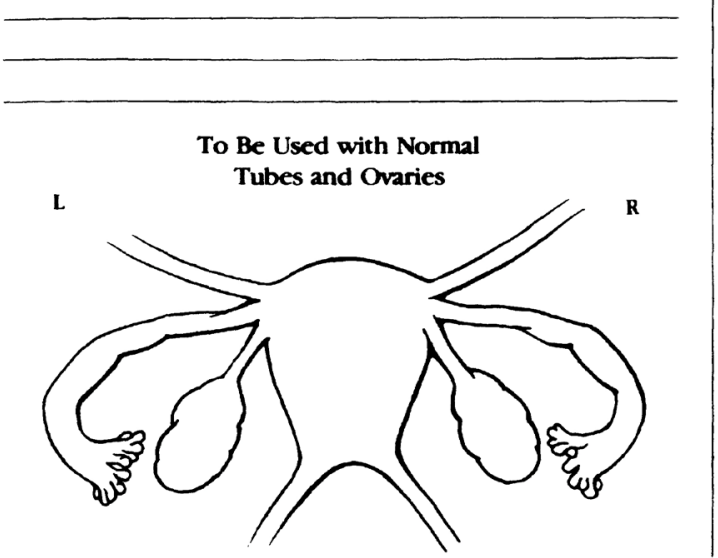

Associated Pathology:

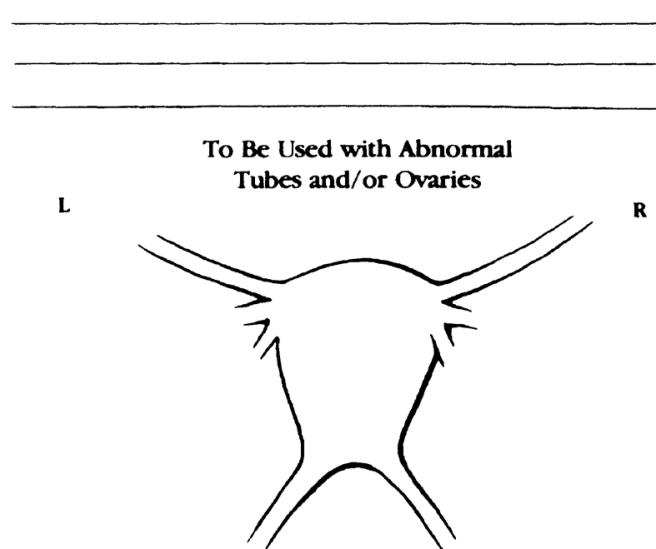

Fig. 1. The revised American Society for Reproductive Medicine classification of endometriosis. This system is the well-known classification of endometriosis. Reproduced from American Society for Reproductive Medicine [8] with permission of Elsevier. 


\section{ENZIAN 2012}

Classification of Deep Infiltrating Endometriosis (according to the Endometriosis Research Foundation, SEF)

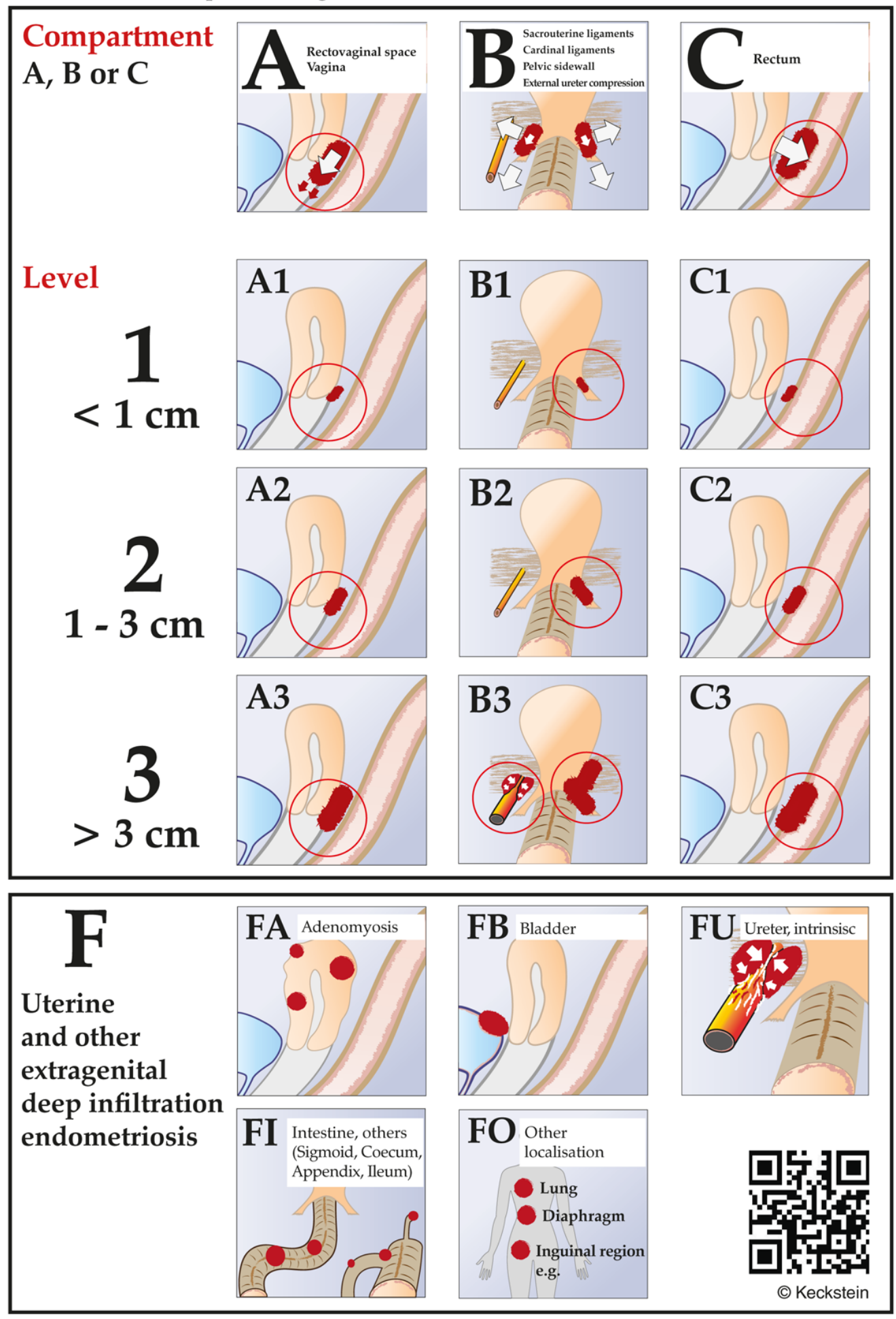

Fig. 2. The ENZIAN staging system for women with deep endometriosis. This system was developed as a supplement to the revised American Society for Reproductive Medicine score, in order to provide detailed descriptions of the retroperitoneal structure. Adapted from 7th Conference of the Stiftung Endometriose Forschung [16] (https://www.endometriose-sef.de/aktivitaeten/klassifikation-enzian/). 
The compartment can be divided into three sections and the severity of each compartment as well as that of the distant lesions, such as diaphragmatic and ureteral invasions, can be described. Second, the ENZIAN classification can be determined by imaging modalities and used for surgical planning. A study has reported that magnetic resonance imaging (MRI) predicts the extent of disease before surgery using ENZIAN score and enables preoperative surgical planning. Di Paola et al. [17] conducted a retrospective study $(\mathrm{n}=115)$ comparing histopathological ENZIAN scores with ENZIAN scores detected by preoperative MRI. The accuracy of the ENZIAN scores detected by preoperative MRI was $95 \%$ with a low false-negative rate of $4 \%$ [17]. Third, disease localization and extent, as described by the revised ENZIAN score, are associated and correlated with the presence and severity of different symptoms [18]. Another researcher has also reported that the ENZIAN classification seems to have a relationship with pain, although general consensus is weak [19].

However, there are several drawbacks. First, the ENZIAN classification has a poor level of international acceptance. It is currently not widely used but mainly used in German-speaking countries. Second, patients may not readily understand the ENZIAN classification because of the complexity of the stage and insufficient knowledge of the pelvic anatomy. If there is a 2-cm unilateral infiltration of endometriosis, the ENZIAN classification expresses it as 'E2b.' This terminology does not accurately convey the severity of the disease to the patient. Third, the ENZIAN score will be inaccurate if the surgical dissection of the deep invasive lesions is incompletely performed, or if image study alone is performed without surgery. Fourth, even if the ENZIAN classification is predicted by imaging modalities, there is insufficient research regarding the usefulness of the classification determined by imaging. Further study is needed to clarify whether the preoperative ENZIAN score can be used for evaluating the surgical feasibility or complete resectability.

\section{Endometriosis fertility index}

The purpose of the development of the EFI system is to predict the pregnancy rate in patients with surgically documented endometriosis who have not attempted to become pregnant with in vitro fertilization (IVF). In 2010, Adamson and Pasta [20] proposed an EFI system based on the data from 579 infertile patients with surgically diagnosed endometriosis (Fig. 3). After EFI was developed, the data of an additional 222 patients were collected for correlation of actual outcomes. The EFI system reflects historical factors such as age, duration of infertility, and previous pregnancies. For pregnancy, proper functioning of the fallopian tube, fimbria, and ovary is required. The functional score indicates whether the embryo is well implanted into the uterus, whether the uterus can provide an early environment for the embryo, or whether the fallopian tubes can pick up the ovum well. The least function score is calculated by evaluating the function of the ovary, fallopian tube, and fimbria for each side, and adding the lowest score on the left and the lowest score on the right. Functional scores are determined by the surgeon and range from 0 to 4 points as follows; absent or nonfunctional as 0 , severe dysfunction as 1 , moderate dysfunction as 2 , mild dysfunction as 3 , and normal as 4. Not only the least functional score, but also other surgical factors such as rASRM total score and endometriosis lesion score of rASRM are included. Finally, The EFI score is calculated by summing the historical and surgical scores, and ranges from 0 to 10 points, with 10 indicating the best prognosis and 0 the worst prognosis.

The EFI system has a clear advantage on predicting pregnancy outcome. The EFI score reflects the pregnancy rate better than the rASRM classification does. According to Zeng et al. [21], the pregnancy rate was $53.6 \%, 36.0 \%, 51.7 \%$, and $41.7 \%$ in rASRM stages I, II, III, and IV, respectively, with no statistically significant difference $(p=0.246)$. However, the pregnancy rate according to the EFI score was observed to be statistically significant at $8.3 \%$ in the group with an EFI score of 0 to 3, $41.2 \%$ for a score of 4 to 7 , and $60.9 \%$ for a score of 8 to $10(p<0.001)$ [21]. Similarly, the EFI score is a more reliable system to predict IVF outcomes in endometriosis patients than the rASRM classification. Wang et al. [22] reported that the IVF outcomes were higher in patients with an EFI score of 6 or higher than in those with a score of 5 or less. However, the EFI system has the following disadvantages. First, the EFI score does not correlate with pain. Second, as the least function score is judged subjectively, the total score can vary by surgeon. To date, there is no study on the assessments of interobserver reliability and intraobserver reproducibility in the EFI system. Third, the EFI score is more complicated to use than the rASRM classification and ENZIAN score, since it requires the calculation and addition of the scores of various categories.

\section{American Association of Gynecological Laparoscopists classification}

In 2007, the AAGL initiated a project to develop a new classification of endometriosis [23]. Thirty endometriosis experts were requested to assign scores ranging from 0 to 10 points on the basis of the importance of each involvement site on the outcomes of pain, infertility, and surgical difficulty. This system contained all the basic information thought to be important in quantifying the 


\section{ENDOMETRIOSIS FERTILITY INDEX (EFI) SURGERY FORM}

\section{LEAST FUNCTION (LF) SCORE AT CONCLUSION OF SURGERY}

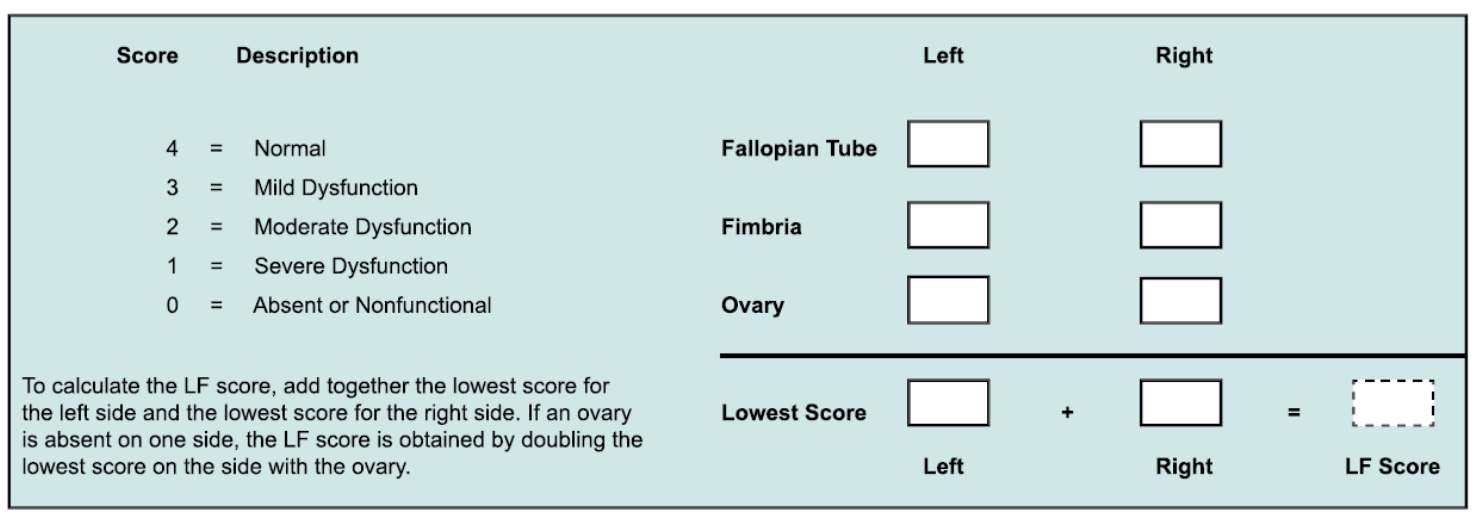

ENDOMETRIOSIS FERTILITY INDEX (EFI)

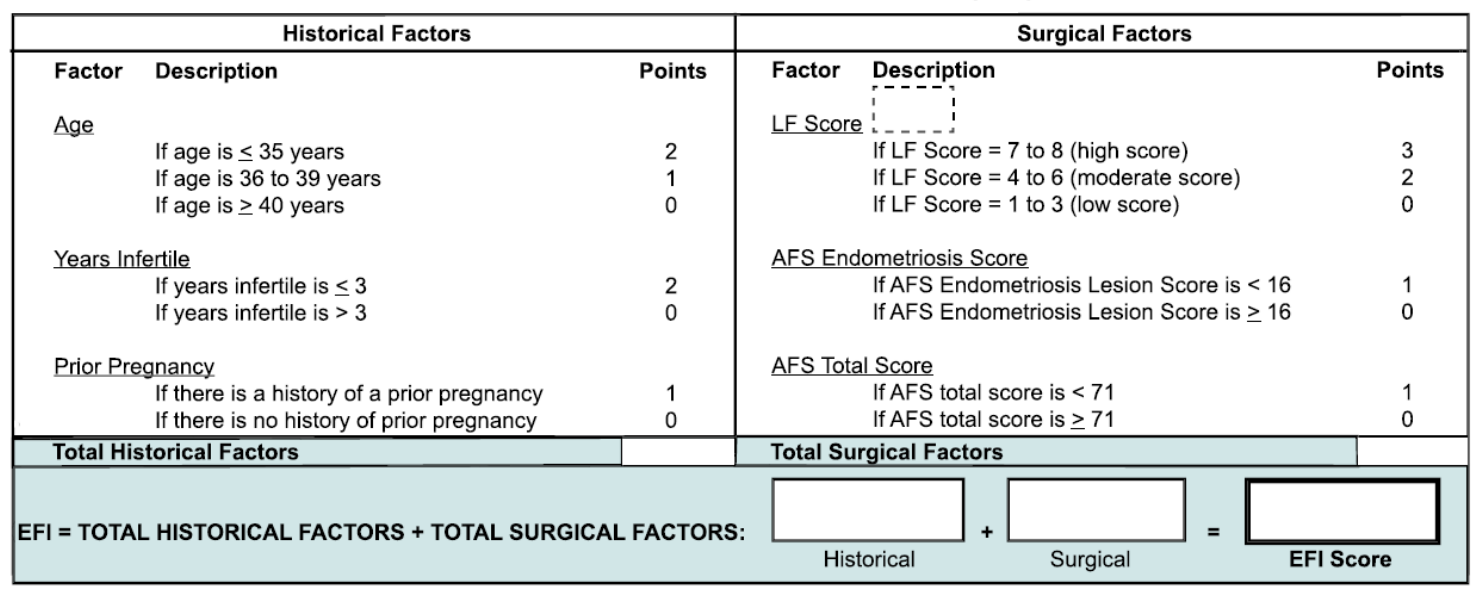

ESTIMATED PERCENT PREGNANT BY EFI SCORE

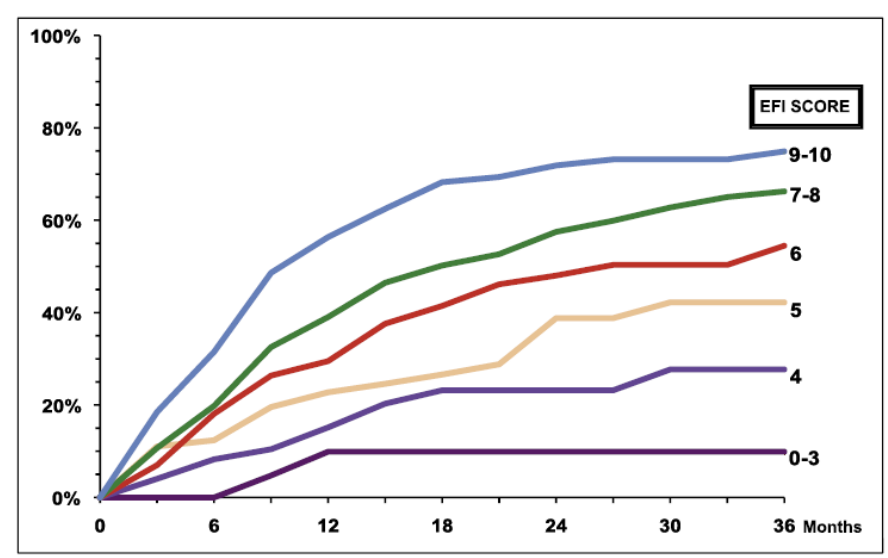

Fig. 3. Endometriosis fertility index (EFI) system. This score predicts the fertility outcome for women who attempt non-in vitro fertilization conception following surgically documented endometriosis. Reproduced from Adamson and Pasta [20] with permission of Elsevier. 
extent of disease in a patient. In addition, surgical difficulties were categorized into four levels: level 1 , excision or desiccation of superficial implants and simple thin avascular adhesions; level 2, stripping of ovarian endometriomas; appendectomy; deep endometriosis not involving the vagina, bladder (not requiring sutures), bowel, or ureter; dense adhesions not involving the bowel and/or the ureter; level 3, dense adhesions involving the bowel and/or ureter; bladder surgery requiring sutures; ureterolysis; bowel surgery without resection (shaving); level 4, bowel resection with end-to-end anastomosis; ureteral reimplantation or anastomosis [24]. For validation of the score system, visual analogue scale scores and infertility history were collected from the patients before surgery. In 2012, the AAGL Special Interest Group reported that the preliminary results presented at the AAGL Las Vegas meeting were encouraging, and that the AAGL classification for endometriosis was verified to be related to pain, infertility, and surgical difficulty. However, the AAGL classification is yet to be fully validated and published, although over 10 years have passed since the classification was first proposed. Further investigation and discussion regarding the AAGL classification are required.

\section{Concerns for the current four classification systems and a need for a novel system in endometriosis}

In addition to the four classifications described in this article, other proposals exist. In the mid-1980s, subtle or non-pigmented lesions were recognized to contain endometrial glands and stroma [25]. Although the significance of subtle lesions is unclear, these may correspond to the initial phase where endometriosis begins. However, it seems difficult to classify these subtle lesions into specific and subdivided systems with the aforementioned classifications. Finally, Koninckx et al. [26] suggested that endometriosis should be classified into subtle, typical, cystic, deep, adenomyotic, and peritoneal pocket lesions in 2011. However, this classification has not been generally accepted and validated.

Each classification reviewed in the present study has its own pros and cons. The biggest challenge of the current classification systems seems to be their poor correlation with symptoms. Symptoms and intensity vary depending on the affected compartment, depth of invasion, and extent of disease; in particular, pain related to endometriosis can be expressed in various phenotypes such as dysmenorrhea, dyspareunia, chronic pelvic pain, and dysuria. The currently existing classification systems have limited research on the relationship between pain and severity of endometriosis. Accordingly, a novel classification system should be developed to better reflect the relationship between pain and endometriosis severity.

In 2011, Abrao and Miller [27] published an article entitled 'Six good reasons for a NEW Endometriosis Classification' They proposed that an ideal classification must meet the following conditions: (1) clearly describe the sites and extent of disease - peritoneal, ovarian, and deep endometriosis, including the bowel, ureter, and bladder; (2) provide a close correlation with the symptoms of endometriosis: pain (dysmenorrhea, dyspareunia, dysuria, and dyschezia) and infertility; and (3) reflect the surgical difficulty encountered relative to the disease location. This is facilitated by the inclusion of increasing radical procedures such as ureterolysis and bowel resection; (4) be user-friendly with tools conducive to supporting a surgeon's busy practice by enabling completion of documentation immediately upon procedure conclusion; (5) be validated for both pain and infertility. With proper validation, this new system can be most useful for therapeutic and prognostic considerations; (6) create a comprehensive universal language that is meaningful for clinical practitioners and researchers alike, thus providing the foundation of collegial collaboration, which ultimately will advance our understanding of the disease.

More recently, the European Society of Human Reproduction and Embryology (ESHRE) published recommendations for the surgical treatment of DIE [28]. A working group of the European Society for Gynaecological Endoscopy, ESHRE and World Endometriosis Society (WES) collaborated to make recommendations for surgical treatment of DIE. They stated the importance of classifying DIE, but also emphasized the limitations of the existing system, specifically with regard to the scoring of the severity of disease. The working group recommended documenting the following information: the location of DIE lesions; uterosacral ligaments, including whether ureters are infiltrated; rectovaginal septum, including involvement of vaginal wall/mucosa; bowel, including involvement of the muscularis layer; bladder, including involvement of muscularis and ureteral ostia; other sides in the pelvis; extrapelvic locations; involvement of the ovaries; the sizes of the lesions; the number of lesions; and the degree of involvement of adjacent organs and structures.

To date, there is no gold standard for the classification of endometriosis. Only expert consensus regarding the current classification systems exists. The WES released a consensus statement on the classification of endometriosis in 2017. International experts from 29 organizations systematically evaluated the classification of endometriosis and reached this consensus [19]. The WES suggested that the following recommendations should be considered in critical situations. All women undergoing surgery must complete rASRM classification to obtain the maximum information 
until better classification is available, and women with DIE must additionally complete ENZIAN classification. EFI must be additionally completed in women who need to consider fertility in future [19].

\section{Conclusion}

To date, no single classification system adequately classifies endometriosis. The rASRM classification is the most widely used and is useful for physicians to explain the severity of endometriosis in simple terms to the patients. The ENZIAN classification describes DIE involving retroperitoneal structures in detail. In addition, ENZIAN classification can probably be determined using imaging modalities and be used for surgical planning. However, external validation of the value of ENZIAN in further studies is needed. EFI score predicts the fertility outcome for women who attempt non-IVF conception following surgically documented endometriosis. Likewise, the EFI score appears to be a reliable system to predict IVF outcomes in endometriosis patients. There is still much to learn and much to do with respect to the classification of this complex and challenging disease. An optimal classification should be established to accurately reflect symptom and disease extents as well as to determine treatment strategies. Furthermore, it would be more ideal if the disease stage and clinical prognosis are accurately predictable using a new classification system without surgical approach.

\section{Acknowledgments}

\section{Conflicts of interest}

No potential conflict of interest relevant to this article was reported.

\section{Author contributions}

Conceptualization and Supervision: YJK, DHL; Formal analysis: all authors; Project administration: DHL; Writing-original draft: SYL; Writing-review \& editing: YJK.

\section{ORCID}

Soo-Young Lee, https://orcid.org/0000-0001-8672-0751

Yu-Jin Koo, https://orcid.org/0000-0002-5114-8000

Dae-Hyung Lee, https://orcid.org/0000-0002-0219-0317

\section{References}

1. Dunselman GA, Vermeulen N, Becker C, Calhaz-Jorge C, D’Hooghe T, De Bie B, et al. ESHRE guideline: management of women with endometriosis. Hum Reprod 2014;29:400-12.

2. Adamson GD, Kennedy S, Hummelshoj L. Creating solutions in endometriosis: global collaboration through the World Endometriosis Research Foundation. J Endometr 2010;2:3-6.

3. Statistics Korea. Outpatient benefits by frequency of disease (total) 2018 [Internet]. Daejeon: Statistics Korea; 2019 [cited 2020 May 8]. http://kosis.kr/statHtml/statHtml.do? orgId =350\&tblId=DT_35001_A084112\&vw_cd = MT_ZTITLE\&list_ id $=350 \_35001 \_6 \&$ seqNo $=$ \&lang_mode $=$ ko\&language $=$ kor \&obj_var_id $=\& \mathrm{itm} \_\mathrm{id}=\&$ conn_path $=$ MT_ZTITLE.

4. Soliman AM, Surrey ES, Bonafede M, Nelson JK, Vora JB, Agarwal SK. Health care utilization and costs associated with endometriosis among women with medicaid insurance. J Manag Care Spec Pharm 2019;25:566-72.

5. Sampson JA. Perforating hemorrhagic (chocolate) cysts of the ovary: their importance and especially their relation to pelvic adenomas of endometrial type ("adenomyoma" of the uterus, rectovaginal septum, sigmoid, etc.). Arch Surg 1921;3:245323.

6. The American Fertility Society. Classification of endometriosis. Fertil Steril 1979;32:633-4.

7. Hasson HM. Classification for endometriosis. Fertil Steril 1981;35:368-9.

8. Revised American Society for Reproductive Medicine classification of endometriosis: 1996. Fertil Steril 1997;67:817-21.

9. Fernando S, Soh PQ, Cooper M, Evans S, Reid G, Tsaltas J, et al. Reliability of visual diagnosis of endometriosis. J Minim Invasive Gynecol 2013;20:783-9.

10. Hornstein MD, Gleason RE, Orav J, Haas ST, Friedman AJ, Rein MS, et al. The reproducibility of the revised American Fertility Society classification of endometriosis. Fertil Steril 1993;59:1015-21.

11. Vercellini P, Trespidi L, De Giorgi O, Cortesi I, Parazzini F, Crosignani PG. Endometriosis and pelvic pain: relation to disease stage and localization. Fertil Steril 1996;65:299-304.

12. Guzick DS, Bross DS, Rock JA. Assessing the efficacy of The American Fertility Society's classification of endometriosis: application of a dose-response methodology. Fertil Steril 1982;38:171-6.

13. Tuttlies F, Keckstein J, Ulrich U, Possover M, Schweppe KW, Wustlich M, et al. ENZIAN-score, a classification of deep infiltrating endometriosis. Zentralbl Gynakol 2005;127:275-81.

14. Haas D, Chvatal R, Habelsberger A, Wurm P, Schimetta W, Oppelt P. Comparison of revised American Fertility Society and ENZIAN staging: a critical evaluation of classifications of endometriosis on the basis of our patient population. Fertil Steril 2011;95:1574-8. 
15. Stiftung Endometriose Forschung. 6th Conference of the Stiftung Endometriose Forschung (Foundation for Endometriosis Research); 2010 Feb 19-21; Weissensee, Austria. Weissensee: Stiftung Endometriose Forschung; 2010.

16. Stiftung Endometriose Forschung. The revised Enzian classification. Consensus meeting, 7th Conference of the Stiftung Endometriose Forschung (Foundation for Endometriosis Research); 2011 Feb 25-27; Weissensee, Austria. Weissensee: Stiftung Endometriose Forschung; 2011.

17. Di Paola V, Manfredi R, Castelli F, Negrelli R, Mehrabi S, Pozzi Mucelli R. Detection and localization of deep endometriosis by means of MRI and correlation with the ENZIAN score. Eur J Radiol 2015;84:568-74.

18. Montanari E, Dauser B, Keckstein J, Kirchner E, Nemeth Z, Hudelist G. Association between disease extent and pain symptoms in patients with deep infiltrating endometriosis. Reprod Biomed Online 2019;39:845-51.

19. Johnson NP, Hummelshoj L, Adamson GD, Keckstein J, Taylor HS, Abrao MS, et al. World Endometriosis Society consensus on the classification of endometriosis. Hum Reprod 2017;32: $315-24$.

20. Adamson GD, Pasta DJ. Endometriosis fertility index: the new, validated endometriosis staging system. Fertil Steril 2010; 94:1609-15.

21. Zeng C, Xu JN, Zhou Y, Zhou YF, Zhu SN, Xue Q. Reproductive performance after surgery for endometriosis: predictive val- ue of the revised American Fertility Society classification and the endometriosis fertility index. Gynecol Obstet Invest 2014; 77:180-5.

22. Wang W, Li R, Fang T, Huang L, Ouyang N, Wang L, et al. Endometriosis fertility index score maybe more accurate for predicting the outcomes of in vitro fertilisation than r-AFS classification in women with endometriosis. Reprod Biol Endocrinol 2013;11:112.

23. Endometriosis Classification Committee; Ad hoc committee of the AAGL. AAGL endometriosis tabulation system. California (CA): Cypress; 2007.

24. Chapron C, Abrao MS, Miller CE. Endometriosis classifications need to be revisited: a new one is arriving. NewsScope 2012; 26(4):9-10.

25. Jansen RP, Russell P. Nonpigmented endometriosis: clinical, laparoscopic, and pathologic definition. Am J Obstet Gynecol 1986;155:1154-9.

26. Koninckx PR, Ussia A, Adamyan L, Wattiez A. An endometriosis classification, designed to be validated. Gynecol Surg 2011; 8:1-6.

27. Abrao MS, Miller CE. An endometriosis classification, designed to be validated. NewsScope 2012;25(4):6.

28. Working group of ESGE, ESHRE, and WES; Keckstein J, Becker CM, Canis M, Feki A, Grimbizis GF, et al. Recommendations for the surgical treatment of endometriosis. Part 2: deep endometriosis. Hum Reprod Open 2020;2020:hoaa002. 